\title{
Quantum Analogue Computing
}

\author{
By Vivien M. Kendon ${ }^{1}$, Kae Nemoto ${ }^{2}$ and William J. Munro ${ }^{2,1}$ \\ ${ }^{1}$ School of Physics and Astronomy, University of Leeds, Leeds LS2 9JT, UK \\ ${ }^{2}$ National Institute of Informatics, 2-1-2 Hitotsubashi, Chiyoda-ku, Tokyo, \\ 101-8430, Japan
}

We briefly review what a quantum computer is, what it promises to do for us, and why it is so hard to build one. Among the first applications anticipated to bear fruit is quantum simulation of quantum systems. While most quantum computation is an extension of classical digital computation, quantum simulation differs fundamentally in how the data is encoded in the quantum computer. To perform a quantum simulation, the Hilbert space of the system to be simulated is mapped directly onto the Hilbert space of the (logical) qubits in the quantum computer. This type of direct correspondence is how data is encoded in a classical analogue computer. There is no binary encoding, and increasing precision becomes exponentially costly: an extra bit of precision doubles the size of the computer. This has important consequences for both the precision and error correction requirements of quantum simulation, and significant open questions remain about its practicality. It also means that the quantum version of analogue computers, continuous variable quantum computers (CVQC) becomes an equally efficient architecture for quantum simulation. Lessons from past use of classical analogue computers can help us to build better quantum simulators in future.

Keywords: quantum information, quantum computation, quantum simulation

\section{Introduction}

It is a quarter of a century since the seminal work of Feynman (1982) and Deutsch (1985) introduced the concept of quantum computation, and fifteen years since Shor (1997) produced an efficient quantum algorithm for factoring large numbers, thereby raising the game for cryptographic schemes by suggesting a quantum computer could break them more easily. Yet working quantum computers are still just toys in a test tube performing calculations a child can do in their head. This is not for want of trying. Spurred on by the seminal work of Shor (1995), Laflamme et al. (1996), Steane (1996), who showed how quantum error correction can be used to protect quantum systems from decoherence for long enough to run a computation, the growth in research has been phenomenal on both theoretical and experimental aspects of the challenge to construct a quantum computer. Steady progress has been made over the intervening years, and there are a plethora of promising architectures on the drawing board and the laboratory bench. There is currently no clear front runner in the pack. We are at a stage of development equivalent to that of classical computers before the advent of silicon chips (a mere fifty years ago). We know a lot about what a quantum computer should be able to do, and what components it needs, but we haven't identified the best materials to build it with, nor what it will be most useful for calculating. 


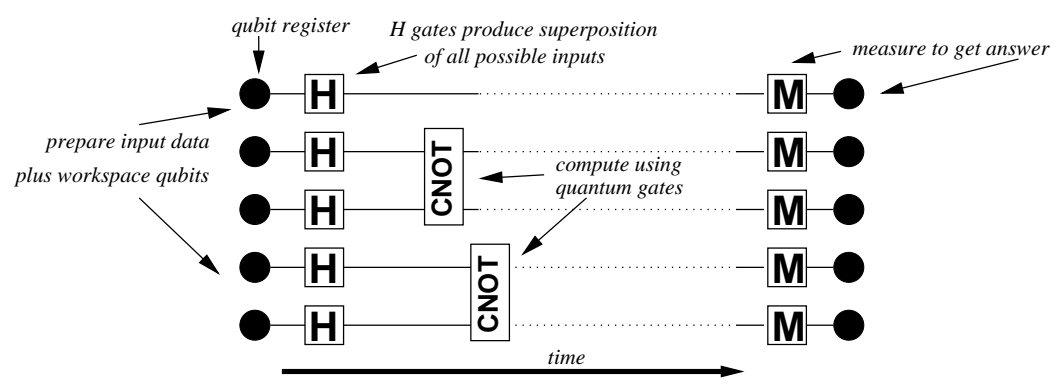

Figure 1. Digital quantum computation (gate model).

\section{Quantum computing}

Feynman and Deutsch both (independently) perceived that a superposition of multiple quantum trajectories looks like a classical parallel computer, which calculates the result of many different input values in the time it takes for one processor to do one input value. Except the quantum system doesn't need a stack of CPUs, the parallel processing comes 'for free' with quantum dynamics, potentially providing a vast economy of scale over classical computation. Of course, there is no 'free lunch'. Despite doing all the calculations in superposition, we can only access one of the results when we measure the final state of the quantum computer. Figure 1 illustrates schematically how a simple gate model quantum computer works.

Programming for a quantum computer is thus trickier than classical programming: you have to include a step to select the answer you want (at least with high probability) out of all the possible results. Quantum programming techniques can be grouped into a few distinct types. Promise problems, like Deutsch and Jozsa (1992), hidden subgroup problems, like Shor (1997) for factoring, search algorithms like Grover (1996), and quantum versions of random walks (see Ambainis (2004) for a review, and Kendon (2006) for a gentle introduction). Some techniques are best understood as a different architecture rather than a different programming method. Minimisation problems, such as finding ground state energies, or satisfiability problems (kSAT) are best tackled using adiabatic quantum computation (Farhi et al. 2000). Adiabatic quantum computation has been proved to be equivalent to the standard gate model of quantum computing (Aharonov et al. 2007), meaning that we will be able to run adiabatic quantum algorithms on any quantum computer.

Many of these potential applications will require very large quantum computers before they gain an advantage over classical computers. For example, the best classical factoring to date (Bahr et al. 2005) can handle a 200 digit number (RSA200 ), which is about 665 bits. For an $n$-bit input number, a basic implementation of Shor's quantum factoring algorithm needs $2 n$ qubits in the QFT register plus $5 n$ qubits for modular exponentiation $=7 n$ logical qubits, so a 665 bit number needs 4655 logical qubits. For a quantum computer of this size, error correction will be essential. How much this increases the number of physical qubits depends on the error rates. The closer to the threshold rates of $10^{-3}$ to $10^{-4}$, the more error correction is necessary (Devitt et al. 2009). These threshold error rates are smaller than any experiment has yet achieved. For low error rates, maybe 20-200 physical qubits per logical qubit are sufficient, while for high error rates (close the threshold) 
it blows up quickly to around $10^{5}$ per logical qubit. This suggests we may need Teraqubit quantum computers to break factoring. While the scaling favours quantum, the crossover point is high.

The picture is rather more promising for using a quantum computer to simulate a quantum system, Fevnman's original inspiration for quantum computation. For example, a system consisting of $N 2$-state quantum particles has $2^{N}$ possible states, and in general, it could be in superposition of all of them. Classical simulation of such a system requires one complex number per state, which amounts to $2^{N+1} \times$ sizeof-double. With 1 Gbyte of memory, a system of $N=26$ of the 2-state quantum particles can be accommodated. The record set by De Raedt et al. (2007) is $N=36$ in 1Terabyte of memory. Each additional particle doubles the amount of memory required. Simulating more than 40 or so qubits in a general superposition state is beyond current classical capabilities. If the system is restricted to only part of its full Hilbert space, not all superpositions are needed, and with appropriately designed methods to take this into account, larger classical simulations are possible (Verstraete et al. 2004). This suggests that quantum computers with upwards of 40 logical qubits could perhaps do something useful for us we can't accomplish with classical simulation, in stark contrast to the Teraqubits required for factoring.

While a classical simulation allows the full quantum state to be tracked throughout, data extraction from quantum simulations is not nearly so easy. Each run provides only a single snapshot of the outcome of a measurement on the superposition state of the system. But methods have been designed for extracting key parameters, such as energy gaps (Wu et al. 2002), or correlation functions (Somma et al. 2002) from quantum simulations. Open problems in the theory of nanoscale superconducting materials are one example where quantum simulation could make an impact on the development of the field.

Quantum simulation has been demonstrated (Somaroo et al. 1999), for small systems using NMR quantum computers. So what is stopping us from building a quantum computer with 40 to 100 qubits tomorrow? Quite simply, this is extremely demanding technology to design and build. It requires single quantum particles to be localised and controlled for long enough to apply hundreds or thousands of precise manipulations to them, singly or in pairs, and then measured individually with high fidelity. And throughout the process, unwanted interactions from nearby material or light must be prevented, or minimised and any remaining effects reversed using error correction techniques. Current state of the art for a quantum simulator is $\lesssim 10$ qubits. Scaling up to 50 with a few hundred manipulations is believable with the steady rate of development the experimentalists have been delivering. And it is believable that at around 50 qubits, a quantum simulator could perform a short computation without needing significant amounts of error correction. Going beyond this without error correction is stretching credibility based on what we know today. But error correction designed for digital quantum computers doesn't work well enough for a quantum simulator. We will elaborate on this point in section 4, after discussing simulation in general, to set the problem in context.

\section{Simulation}

Computer simulations play an important role in most areas of science. Numerical methods kick in wherever analytic calculations become too complicated or 


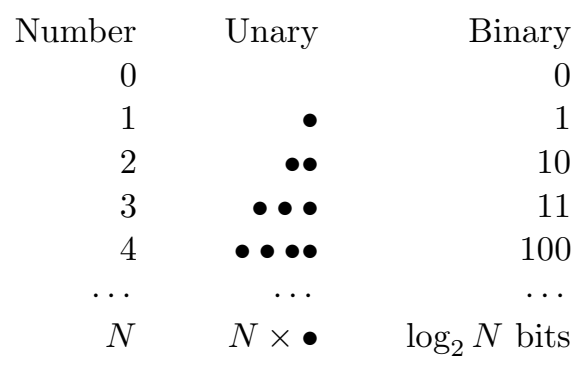

Figure 2. Binary counting needs exponentially less memory than unary.

intractable, which can happen even for simple systems, like three bodies moving under their mutual gravitational interactions. We use computer simulation to test our models of the real world, by calculating in detail what they predict, then comparing this with our experimental observations. We don't expect perfect agreement, both experiments and computations have limited precision, and we may know the model is only an approximation (to simplify the computation). If our calculations and observations agree as well as we anticipate, this is evidence our models are good, and hence we understand at some level how the system works. We may then use our computer simulations to predict things we haven't yet observed, or provide more details of processes that are hard to observe by experiment. We may even simulate systems that can't, or don't, exist in nature, to better understand why nature is the way it is.

That computation of any sort works in a useful way is not trivial. An accurate calculation of the trajectory of a space probe that takes five years to reach its destination is crucial: we cannot just launch many test probes and see where they end up in order to work out which trajectory we want. One key difference between a classical physical system and a computer simulation of it is that we represent it using binary numbers ( 0 and 1$)$ in a register in the computer. This is like the difference between counting on your fingers and writing down the number 8 . Eight has just one symbol in place of your eight fingers. Eight people have 64 fingers between them and that only took two symbols to write down. This makes for a huge saving in the amount of memory a computer needs compared to the size of the physical system, see figure 2. Binary numbers mean counting using base two, whereas we usually use base ten. The saving in memory is huge whichever base we use, but the inner workings of the computer are easier to arrange using binary.

A second important reason why computer simulations work depends on the complexity of the model compared with the complexity of the system being studied. Stars and planets are highly complex systems, but we don't need to model most of the details to predict to very high precision how the planet will orbit the star. Gravitation is a supremely simple theory compared with systems it can correctly describe the behaviour of. This is part of the way our world appears to be, described as the "unreasonable effectiveness of mathematics" by Wigner (1960). More discussion of simulating complex classical systems, and how to apply quantum computers to this task, can be found in Harris and Kendon (2010), here we are specifically interested in simulating quantum systems. 


\section{Efficiency and precision}

For practical purposes, an efficient computation is one that gives answers in relatively short human time scales (seconds, minutes, hours or days) at a cost we can afford to pay. Computer scientists make quantitative comparisons between different algorithms by associating a cost with each step of the algorithm and with the amount of memory required to hold the data. This abstracts the idea that physical computers have a limited size (memory) and maximum rate of elementary calculation steps per unit time. In most cases, 'efficient' means the algorithms use resources that scale as a simple polynomial (like $N^{2}$ ) of the size $N$ of the problem.

If we could efficiently simulate a quantum algorithm on a classical computer, we would immediately have a classical algorithm that is sufficiently good to render the quantum algorithm superfluous. To prove an algorithmic speed up for a quantum algorithm, we have to prove that no classical algorithm of any type can do as well: such proofs are in general very difficult. Shor's algorithm (Shor 1997) is only the 'best known' algorithm for factoring, there is no proof something classical and faster can't be found in future.

Just as we can't usually make a perfect measurement that determines exactly how long or heavy something is, we also generally have to represent numbers in a computer to a fixed precision. As the computation proceeds, the errors due to the fixed precision slowly grow. For very long computations, they can overwhelm the answer and render it useless. The accuracy we require for our answer thus places another demand on how much memory and time we need to perform our computation. Starting out with a higher precision to represent our numbers allows high precision for the answer. If we represent our numbers as floating point with an exponent, e.g., $0.1011101101 \times 2^{4}$, then for an error of size $\varepsilon$, the number of bits (binary digits) of precision is $\log _{2}(1 / \varepsilon)$. For the example just given, which has ten bits of precision, $\varepsilon=2^{-10}$. Efficient algorithms require resources (time and memory) that scale as $\log _{2}(1 / \varepsilon)$. Algorithms running on digital computers, that store their numbers in this binary format, usually have this logarithmic scaling for precision. However, for computers that store information in a unary encoding, such as quantum simulators, the errors will scale as $1 / \varepsilon$, thus requiring exponentially more resources for the same increase in precision as their binary encoded counterparts.

Approximating real numbers to a fixed precision introduces errors into calculations that grow larger as the computation proceeds. If the computation requires many steps, it may be necessary to use a higher precision to avoid the errors swamping the final answer. As well as errors arising from the limited precision, the operation of the computer itself may be imperfect and introduce random errors. In our present day classical computers, such errors are so unlikely to happen we can forget about them. In contrast, error correction for digital quantum computation will be essential beyond about 100 qubits and 100 computational steps.

\section{Quantum simulation of quantum systems}

The main reason why classical simulations of quantum systems are not efficient is because the memory required scales exponentially in the system size, due to the exponential number of quantum superpositions. This can be solved by using the efficient storage achieved by quantum computers. Mapping the Hilbert space of the 


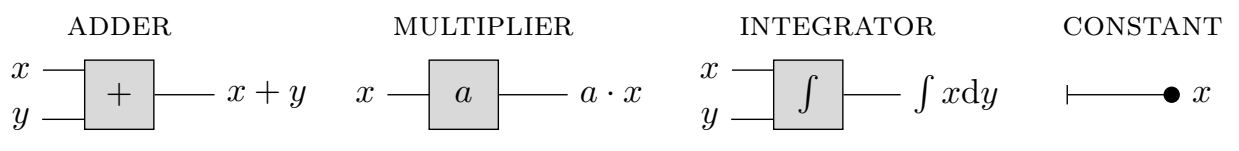

Figure 3. GPAC operation set.

system directly onto the Hilbert space of the quantum computer gives us efficient memory use. We also require that a quantum simulator can run using an efficient number of time steps. Llovd (1996) showed that the Hamiltonian evolution of the quantum system can be decomposed into a sequence of standard Hamiltonians by using the Trotter approximation. Each standard Hamiltonian is applied for a short time to build up the desired evolution. The number of steps required scales polynomially in the accuracy, so this part of the algorithm is also efficient.

However, any small errors or imperfections in the operation of the quantum simulator will affect the result in a linear rather than logarithmic way, because the system is mapped directly onto the quantum simulator, rather than being binary encoded into numbers in qubit registers. Consequently, we will need exponentially more resources to correct for these errors. This raises the prospect of crippling error correction requirements for practical quantum simulators (Brown et al. 2006).

Quantum simulation has much in common with analogue computation in the way the data is encoded. It shares the problem that an extra bit of precision requires a doubling of the resources. However, this suggests two avenues for the development of quantum simulation that have not yet been well-explored. Firstly, looking back to the era of analogue computation can teach us lessons for how to make quantum simulation practically useful, despite the unfavorable scaling of the resources. Secondly, we can consider quantum versions of analogue computation for quantum simulation architectures. We discuss both these ideas in the next sections.

\section{Analogue computing}

Despite the exponential saving in memory gained through binary encoding, the earliest computers didn't use it. From the invention of the astrolabe for plotting the heavens in around $200 \mathrm{BC}$, through the slide rule, and mechanical differential analyser (Thomson 1876), these computational devices represented the quantity they were computing as the size of some part of the apparatus. If you wanted a more accurate answer, you had to build a larger device: for another bit or precision, it would need to be twice the size. Yet, for the problems these machines were designed to solve, they were very effective in practice.

Shannon (1941) provided a theoretical model, the General Purpose Analogue Computer (GPAC), which is a mathematical description of the differential analyser. The GPAC consists of a set of nonlinear boxes, connected by their inputs and outputs. Four basic operations are sufficient, shown in figure 3. There are a few rules for how the boxes can be connected. Each output of a box goes to at most one input; inputs cannot be interconnected, i.e., there is no "splitter" to allow the same input to go to more than one box; similarly, outputs cannot be interconnected. It has been proved by Pour-El (1978), and Lipshitz and Rubel (1981) that the set of functions generated this way is the set of differentially algebraic functions. In other words, we can solve any ordinary differential equation with this type of device, 
given appropriate boundary conditions as part of the input. This abstract notion of computation can be realised in many different physical systems, such as water in pipes or electronic circuits. Such systems were quite common in university science departments before digital computers became widespread.

There have been a number of suggestions for how to expand the model by adding more operations to the available set e.g., Rubel (1993), Graca (2004), Mills (2008). These are mathematical extensions and it isn't clear whether they can be physically constructed, raising the question of just exactly what we can in practice compute given the way the physical world works. Rubel (1981) observed that there are simple differential equations that are universal in the sense that they can approximate any other differential equation to arbitrary accuracy. In practice, these probably aren't useful for computation, attempts to build a circuit that implements such a differential equation turn out to be completely unstable (Mills 2009). While Rubel (1989) proved that a classical digital computer can efficiently simulate an analogue computer, the reverse question of whether an analogue computer can simulate a digital computer efficiently is open.

Analogue computers might still provide a useful function in our digital world: for certain calculations they are extremely fast. When a specific task is required repeatedly (e.g., video rendering in real time), a dedicated analogue "chip" might outperform a digital circuit.

\section{Continuous variable quantum computing}

There is a fully quantum version of analogue computing, usually known as continuous variable quantum computing (CVQC) and first described by Lloyd and Braunstein (1999). The information is encoded in the eigenstates of a continuous-spectrum operator such as position $\hat{x}$ or momentum $\hat{p}$. Computations are carried out by manipulating the physical states. Position and momentum are a conjugate pair of operators, which are orthogonal in the sense that $[\hat{x}, \hat{p}]=i$ up to a real normalisation constant. They act on the Hilbert Space $L_{2}(\mathbb{R})$, the space of square-integrable functions over $\mathbb{R}$ (square-integrable is important as it corresponds to being normalisable). To perform a quantum computation, we create an initial state, evolve the state in a prescribed way by applying an appropriate Hamiltonian to the state, and then perform a measurement from which we can extract the results.

Universal computation can be done in CVQC using a small set of elementary operations. Any Hamiltonian can be written as a Hermitian polynomial in the position and momentum operators $\hat{x}$ and $\hat{p}$, and we can generate any Hermitian polynomial in $\hat{x}$ and $\hat{p}$ by using the following set of operators (Lloyd and Braunstein 1999):

1. simple linear operations, $\{ \pm \hat{x}, \pm \hat{p}\}$, and quadratic, e.g., $\hat{x}^{2}+\hat{p}^{2}$,

2. a non-linear operation at least cubic i.e., $\hat{x}^{3}$ or $\hat{p}^{3}$, the Kerr Hamiltonian $H_{\text {Kerr }}=\left(\hat{x}^{2}+\hat{p}^{2}\right)^{2}$, may be easier experimentally,

3. an interaction to couple two modes together, e.g., the beam splitter operation.

It is easy to see how this works when the continuous quantity is the position, $x$ (say). After evolving the state with some Hamiltonian, measuring the new position 
gives us the answer (or one possible answer out of a superposition or distribution). But the Heisenberg uncertainty principle means that if we know the position exactly, the momentum is totally undetermined. This asymmetry in the uncertainty is known as squeezing, and using a single quadrature like this requires infinitely squeezed states, which are unphysical. Experimentally, we can't even get a good approximation of an infinitely squeezed state, so for practical purposes, we need to choose a different way to encode our information, and the obvious choice is to use Gaussian states, which have the uncertainty spread between the two quadratures $x$ and $p$. The nonlinear operation in our CVQC will evolve the state into something that is no longer a Gaussian state: if it didn't, the whole computation would be efficiently classically simulatable (Bartlett et al. 2002). This corresponds to having a superposition or distribution of different answers: when we make a measurement, we will interpret the outcome as a Gaussian state from which we can extract the result of out computation.

Implementing CVQC in a real system may require some modification to the set of elementary gates, to adapt it to what is experimentally practical, for an example, see Wagner et al. (2010). While continuous variables are a practical choice for many quantum communications tasks (see Braunstein and van Loock (2005) for a review), very little has been developed for CVQC. In particular, it shares the unfavorable scaling of resources with precision that classical analogue and quantum simulation have, and standard error correction results for digital quantum computation don't apply (Niset et al. 2009).

\section{CVQC for quantum simulation}

For quantum simulation, where the digital advantage of binary encoding is already lost, CVQC deserves a critical second look. Many quantum systems are naturally continuous: for these a CVQC quantum simulation would be especially appropriate. Experimental systems suitable for CVQC can be developed from many of the architectures currently proposed for digital quantum computing: whenever qubits are coupled using some sort of field mode, this can be inverted by regarding the field mode as the continuous variable and the qubits as the control system. Wagner et al. (2010) describes how the micromaser can be used this way, and the photonic module of Devitt et al. (2007) is also promising. Squeezing is one of the elementary operations we need in CVQC when encoding with Gaussian states, and we can use the degree of squeezing to estimate how large our system needs to be to beat classical simulation. Suzuki et al. (2006) have achieved $7 \mathrm{~dB}$ of squeezing in optical experiments. For one mode, $7 \mathrm{~dB}$ of squeezing corresponds to $2-3$ bits of precision i.e., around 5 distinguishable outcomes when the state is measured. With arbitrary perfect coupling, going beyond what we can simulate classically corresponds to more than 17 modes coupled together, since the combination takes us above 40 equivalent (qu)bits. Experimentally, nine modes have been combined in CV error correction by Aoki et al. (2009), though this stays within Gaussian states and is thus still efficiently classically simulatable. This is a very rough estimate, because evolving the system accurately in a classical simulation would in practice require higher precision to produce the required final precision for the output. On the other hand, we can't achieve arbitrary coupled states with current technology. But it does give a feel for the potential for CVQC quantum simulation.

Article submitted to Royal Society 


\section{The future of quantum simulation}

The goal of building a quantum computer large enough to solve problems beyond the reach of the classical computational power available to us, is coming closer. The first useful application for quantum computers is likely to be simulation of quantum systems. Small quantum simulations have already been demonstrated, and useful quantum simulations - that solve problems inaccessible to classical computation - require less than a hundred qubits and may be viable without error correction. While the theoretical underpinnings of digital computation are well developed, there are significant gaps in our understanding of analogue computing theory, and these are in turn slowing the progress of CVQC and quantum simulation. Investment in developing the missing theory is as crucial for the future development of quantum technology as experimental progress in quantum coherence and quantum control.

We thank Katherine Brown, Simon Devitt, Mark Everitt, Martin Jones, and Rob Wagner for useful and stimulating discussions. Thanks to Mark Everitt and Rob Wagner for drafts of figure 3. VK is funded by a Royal Society University Research Fellowship. WM and KN acknowledge funding from the EU project HIP. This work was supported by a UK Royal Society International Joint Project Grant.

\section{References}

Aharonov, D., van Dam, W., Kempe, J., Landau, Z., Lloyd, S., Regev, O., 2007. Adiabatic quantum computation is equivalent to standard quantum computation. SIAM Journal on Computing 37, 166, arXiv:quant-ph/0405098.

Ambainis, A., 2004. Quantum walk algorithms for element distinctness. In: 45th Annual IEEE Symposium on Foundations of Computer Science, Oct 17-19, 2004. IEEE Computer Society Press, Los Alamitos, CA, pp. 22-31.

Aoki, T., Takahashi, G., Kajiya, T., ichi Yoshikawa, J., Braunstein, S. L., van Loock, P., Furusawa, A., 2009. Quantum error correction beyond qubits. Nature Physics 5, 541.

Bahr, F., Boehm, M., Franke, J., Kleinjung, T., 2005. Factored RSA200 on 9th May 2005. URL http://www. loria.fr/ zimmerma/records/factor.html

Bartlett, S., Sanders, B., Braunstein, S. L., Nemoto, K., 2002. Efficient classical simulation of continuous variable quantum information processes. Phys. Rev. Lett. 88, 097904, arXiv:quant-ph/0109047

Braunstein, S. L., van Loock, P., 2005. Quantum information with continuous variables. Rev. Mod. Phys. 77, 513-578, arXiv:quant-ph/0410100v1.

Brown, K. R., Clark, R. J., Chuang, I. L., 2006. Limitations of quantum simulation examined by simulating a pairing hamiltonian using nuclear magnetic resonance. Phys. Rev. Lett. 97, 050504, arXiv:quant-ph/0601021.

De Raedt, K., Michielsen, K., De Raedt, H., Trieu, B., Arnold, G., Richter, M., Lippert, T., Watanabe, H., Ito, N., 2007. Massive parallel quantum computer simulator. Comp. Phys. Comm. 176, 127-136, arXiv:quant-ph/0608239v1.

Deutsch, D., 1985. Quantum-theory, the Church-Turing principle and the universal quantum computer. Proc. R. Soc. Lond. A 400 (1818), 97-117.

Deutsch, D., Jozsa, R., 1992. Rapid solutions of problems by quantum computation. Proc. Roy. Soc. Lon. A 439, 553.

Devitt, S. J., Greentree, A. D., Ionicioiu, R., O'Brien, J. L., Munro, W. J., Hollenberg, L. C. L., 2007. Photonic module: An on-demand resource for photonic entanglement. Phys. Rev. A 76, 052312.

Devitt, S. J., Nemoto, K., Munro, W. J., 2009. The idiots guide to quantum error correction. arXiv:0905.2794v2 [quant-ph]. 
Farhi, E., Goldstone, J., Gutmann, S., Sipser, M., 2000. Quantum computation by adiabatic evolution, arXiv:quant-ph/0001106.

Feynman, R. P., 1982. Simulating physics with computers. Int. J. Theor. Phys. 21, 467.

Graca, D. S., 2004. Some recent developments on Shannon's GPAC. Math. Log. Quart. $50(4-5), 473-485$.

Grover, L. K., 1996. A fast quantum mechanical algorithm for database search. In: Proc. 28th Annual ACM STOC. ACM, NY, p. 212, arXiv:quant-ph/9605043.

Harris, S., Kendon, V., 2010. Quantum-assisted biological modelling. Also submitted to PTRS visions issue.

Kendon, V., 2006. A random walk approach to quantum algorithms. Phil. Trans. Roy. Soc. A 364, 3407-3422.

Laflamme, R., Miquel, C., Paz, J. P., Zurek, W. H., 1996. Perfect quantum error correcting code. Phys. Rev. Lett. 77, 198-201.

Lipshitz, L., Rubel, L. A., 1981. A differentially algebraic replacement theorem, and analog computability. Proc. Amer. Math. Soc. 99, 367-372.

Lloyd, S., 1996. Universal quantum simulators. Science 273, 1073-1078.

Lloyd, S., Braunstein, S. L., 1999. Quantum computation over continuous variables. Phys. Rev. Lett. 82, 1784, arXiv:quant-ph/9810082 1.

Mills, J. W., 2008. The nature of the extended analog computer. Physica D: Nonlinear Phenomena 237 (9), 1235-1256.

Mills, J. W., 2009. Private communication.

Niset, J., Fiurasek, J., Cerf, N. J., 2009. A no-go theorem for gaussian quantum error correction. Phys.Rev.Lett. 102, 120501.

Pour-El, M., 1978. Abstract computability and its relation to the general purpose analog computer, etc. Trans. Amer. Math. Soc. 199, 1-28.

Rubel, L. A., 1981. A universal differential equation. Bull. Amer. Math. Soc. 4, 345-349.

Rubel, L. A., 1989. Digital simulation of analogue computation and Church's thesis. J. Symbolic Logic 54, 1011-1017.

Rubel, L. A., 1993. The extended analog computer. Adv. in Appl. Maths. 14 (1), 39-50.

Shannon, C., 1941. Mathematical theory of the differential analyzer. J. Math. Phys. Mass. Inst. Tech. 20, 337-354.

Shor, P. W., 1995. Scheme for reducing decoherence in quantum computer memory. Phys. Rev. A 52, R2493.

Shor, P. W., 1997. Polynomial-time algorithms for prime factorization and discrete logarithms on a quantum computer. SIAM J. Sci Statist. Comput. 26, 1484.

Somaroo, S. S., Tseng, C. H., Havel, T. F., Laflamme, R., Cory, D. G., 1999. Quantum simulations on a quantum computer. Phys. Rev. Lett. 82, 5381-5384.

Somma, R., Ortiz, G., Gubernatis, J. E., Knill, E., La flamme, R., April 2002. Simulating physical phenomena by quantum networks. Phys. Rev. A 65 (4), 042323.

Steane, A., 1996. Multiple particle interference and quantum error correction. Proc. Roy. Soc. Lond. A 452, 2551, arXiv:quant-ph/9601029.

Suzuki, S., Yonezawa, H., Kannari, F., Sasaki, M., Furusawa, A., 2006. 7 dB quadrature squeezing at $860 \mathrm{~nm}$ with periodically-poled $\mathrm{KTiOPO}_{4}$. Appl. Phys. Lett. 89, 061116.

Thomson, W., 1876. Mechanical integration of the general linear differential equation of any order with variable coeffcients. Proc. Roy. Soc. 24, 271-275.

Verstraete, F., Porras, D., Cirac, J. I., 2004. Density matrix renormalization group and periodic boundary conditions: A quantum information perspective. Phys. Rev. Lett. 93, 227205.

Wagner, R. C., Everitt, M. S., Jones, M. L., Kendon, V. M., 2010. Universal continuous variable quantum computation in the micromaser. In preparation.

Wigner, E., 1960. The unreasonable effectiveness of mathematics in the natural sciences. Communications on Pure and Applied Maths. 13 (1), 1-14.

Wu, L. A., Byrd, M. S., Lidar, D. A., July 2002. Polynomial-time simulation of pairing models on a quantum computer. Phys. Rev. Lett. 89 (5), 057904. 